\title{
Ideação suicida em adolescentes em situação de vulnerabilidade social
}

\author{
Suicidal ideation in adolescents in the situation of social vulnerability \\ Ideación de suicidio en adolescentes en situación de vulnerabilidad social
}

Recebido: 09/02/2021 | Revisado: 13/02/2021 | Aceito: 16/02/2021 | Publicado: 25/02/2021

Bruna Olivia Santos da Silva

ORCID: https://orcid.org/0000-0003-0555-1218

Centro de Fonoaudiologia de Pernambuco, Brasil

E-mail: bruna.olivia@live.com

Marina Araújo Rosas

ORCID: https://orcid.org/0000-0002-5666-7133

Universidade Federal de Pernambuco, Brasil

E-mail: marina.rosas@ufpe.br

Keise Bastos Gomes da Nóbrega

ORCID: https://orcid.org/0000-0002-5837-8183

Universidade Federal de Pernambuco, Brasil

E-mail: keise.nobrega@ufpe.br

Manuela Martins da Silva

ORCID: https://orcid.org/0000-0003-2022-8347

Universidade Federal de Pernambuco, Brasil

E-mail: manuela.msilva@ufpe.br

Maria Gisele Cavalcanti de Oliveira

ORCID: https://orcid.org/0000-0001-5096-0075

Universidade Federal de Pernambuco, Brasil

E-mail: giselecavalcanti1@hotmail.com

Juliana da Silva Cajueiro

ORCID: https://orcid.org/0000-0002-3907-6534 Clínica Espaço Integrar, Brasil

E-mail: kjjuliana@hotmail.com

Mayara Silva Gadelha

ORCID: https://orcid.org/0000-0002-1127-0298 Instituição Novo Rumo, Brasil

E-mail: mhayaragadelha@gmail.com

Naianna Ribeiro Mocelin Dos Santos

ORCID: https://orcid.org/0000-0003-1652-1081 Hospital das Clínicas, Brasil

E-mail: naiannarsantos@gmail.com

Sayonara Queiroz Coelho

ORCID: https://orcid.org/0000-0001-5847-5269 Caps ad CPTRA, Brasil

E-mail: sayonara_queiroz@hotmail.com

Mariza Maria Fabrício

ORCID: https://orcid.org/0000-0002-3141-7074

Banca de Estudos Arte de Criar, Brasil

E-mail: marizamfabricio@hotmail.com

Ivo de Andrade Lima Filho

ORCID: https://orcid.org/0000-0001-6381-4819

Universidade Federal de Pernambuco, Brasil E-mail: ivo.limafo@ufpe.br

\begin{abstract}
Resumo
Objetivo: Identificar entre adolescentes em situação de vulnerabilidade social os que apresentam ideação suicida. Método: Estudo de abordagem quantitativa, corte transversal e descritivo, realizado com adolescentes, com idades 12 a 18 anos, em situação de vulnerabilidade social e façam parte de uma Organização Não Governamental. Para a coleta de dados foram utilizados os instrumentos: questionário estruturado e Escala de Avaliação do Risco de Suicídio de Columbia. Resultados: A maioria dos adolescentes é do sexo feminino, vivem em famílias com média de cinco pessoas, com renda de até um salário mínimo. Dentre os entrevistados 17 adolescentes afirmaram que nunca desejaram estar morto ou nunca pensaram em se matar, enquanto, 13, revelaram a presença de ideação suicida. Todos os adolescentes entrevistados vivem em famílias com média de cinco pessoas, com renda de até um salário mínimo e mais de $50 \%$ dos adolescentes responderam que seus pais são casados ou têm uma união estável. A maior parte dos responsáveis possui ensino fundamental incompleto e $15 \%$ desses estão em situação de desemprego. Conclusão:
\end{abstract}


Frente à gravidade da temática, é necessário pensar em intervenções eficazes que possam detectar precocemente a presença de ideação suicida nos adolescentes.

Palavras-chave: Adolescência; Saúde mental; Vulnerabilidade social; Ideação suicida.

\begin{abstract}
Objective: To identify adolescents in situations of social vulnerability who have suicidal ideation. Method: Quantitative, cross-sectional and descriptive study, carried out with adolescents, aged 12 to 18 years, in a situation of social vulnerability and who are part of a Non-Governmental Organization. For data collection, the following instruments were used: structured questionnaire and Columbia Suicide Risk Assessment Scale. Results: Most adolescents are female, live in families with an average of five people, with an income of up to one minimum wage. Among the interviewees, 17 adolescents stated that they never wished to be dead or never thought about killing themselves, while 13 revealed the presence of suicidal ideation. All adolescents interviewed live in families with an average of five people, with an income of up to one minimum wage and more than $50 \%$ of the adolescents answered that their parents are married or have a stable union. Most of those responsible have incomplete elementary education and $15 \%$ of them are unemployed. Conclusion: In view of the seriousness of the theme, it is necessary to think of effective interventions that can detect the presence of suicidal ideation in adolescents early.
\end{abstract}

Keywords: Adolescence; Mental health; Social vulnerability; Suicidal ideation.

\begin{abstract}
Resumen
Objetivo: Identificar adolescentes en situación de vulnerabilidad social que tengan ideación suicida. Método: Estudio cuantitativo, transversal y descriptivo, realizado con adolescentes de 12 a 18 años, en situación de vulnerabilidad social y que forman parte de una Organización No Gubernamental. Para la recolección de datos se utilizaron los siguientes instrumentos: cuestionario estructurado y Escala de Evaluación de Riesgo de Suicidio de Columbia. Resultados: La mayoría de los adolescentes son mujeres, viven en familias con un promedio de cinco personas, con un ingreso de hasta un salario mínimo. Entre los entrevistados, 17 adolescentes manifestaron que nunca desearon estar muertos o nunca pensaron en suicidarse, mientras que 13 revelaron la presencia de ideación suicida. Todos los adolescentes entrevistados viven en familias con un promedio de cinco personas, con un ingreso de hasta un salario mínimo y más del $50 \%$ de los adolescentes respondieron que sus padres están casados o tienen una unión estable. La mayoría de los responsables tienen educación primaria incompleta y el 15\% de ellos están desempleados. Conclusión: Dada la gravedad del tema, es necesario pensar en intervenciones efectivas que puedan detectar precozmente la presencia de ideación suicida en adolescentes.
\end{abstract}

Palabras clave: Adolescencia; Salud mental; Vulnerabilidad social; Ideación suicida.

\title{
1. Introdução
}

A adolescência é uma fase entre a infância e a vida adulta em que ocorrem diversas modificações caracterizadas pelos impulsos do desenvolvimento físico, mental, emocional, sexual e social. Nesta fase ocorre uma busca de identidade com objetivo de alcançar expectativas culturais de uma determinada sociedade (Eisenstein, 2005).

Algumas transformações permeiam a vida social do indivíduo nesta etapa de construção da identidade. A representação social da adolescência passou a ser vinculada a um período de incertezas, caracterizado por um espaço circunscrito entre a idade infantil e a idade adulta. É durante esse período de instabilidade que se configura uma diversidade de situações e experiências pelas quais o adolescente irá se confrontar até chegar à idade adulta. A busca pela emancipação e independência, no entanto, será fortemente marcada pelos condicionantes externos que adentram a sociedade e o momento histórico em que o adolescente estiver situado (Quiroga, 2013).

Algumas características psicossociais presentes nessa fase são introversão, alternando com audácia, timidez, descoordenação, urgência, desinteresse ou apatia, que se sucedem ou são concomitantes com conflitos afetivos, crises religiosas, intelectualizações e postulações filosóficas (Borges, 2006).

$\mathrm{Na}$ adolescência existe uma situação que obriga o indivíduo a reformular os conceitos que tem acerca de si mesmo e que o levam a abandonar sua autoimagem infantil e a projetar-se no futuro de sua vida adulta, gerando dúvidas acerca dessa nova fase. Esse conflito da adolescência deve ser tomado como um processo universal de troca, de desprendimento, mas que será influenciado por conotações externas peculiares de cada cultura, que o favorecerão ou dificultarão, o desenvolvimento saudável (Borges, 2006). 
Dependendo das circunstâncias de vida que esse adolescente esteja inserido, eles estão expostos a situações de violência, ao uso de drogas e a experiências relacionadas a privações no que diz respeito a ordem afetiva, cultural e socioeconômica desfavorecendo o progresso biopsicossocial. Concerne ressaltar que a disposição de acesso aos meios de comunicação, a escolarização, a disponibilidade de enfrentar barreiras culturais e estar livre de repressões violentas ou poder defender-se delas são componentes importantes para avaliar uma condição de maior ou menor vulnerabilidade social de um indivíduo ou de coletivos (Pessalacia, 2010).

Existem alguns fatores que torna o adolescente mais vulnerável a sofrer problemas na área da saúde mental, como: os individuais (sexo; idade; características psicológicas como autoestima, autoconfiança e determinação), os familiares (história de problemas de saúde mental, especialmente materna; problemas de álcool/drogas; violência física, psicológica e sexual; violência entre os pais; perdas por morte; separação dos pais),os socioculturais (pobreza, violência no contexto social, apoio/suporte social) e os biológicos (mudanças corporais da puberdade, como: altura, forma e sexualidade de adultos), sendo necessário um entendimento de como esses fatores articulam-se entre si e influenciam no comportamento e na saúde desse adolescente (Avanci, 2007; Schoen-Ferreira, 2010).

Vale salientar que, segundo Musa et al., (1997), os adolescentes compõem a faixa etária com maior mobilização em relação a atenção a comportamentos de risco, em relação à saúde. Nesse período de vida que as variações de humor e os conflitos emocionais são mais e característicos, trazendo alguns problemas como a ideação suicida, que se refere ao pensamento e planejamento do ato de uma tentativa de suicídio, podendo ser um primeiro passo para a execução (Pessalacia, 2010; Souza, 2010).

O desejo de morrer (pensamento suicida) presente em alguns momentos da adolescência não é considerado anormal, pois estes pensamentos fazem parte do processo de desenvolvimento da passagem da infância para a adolescência, uma vez que nessa fase o adolescente lida com problemas existenciais e busca compreender a vida. Deste modo, o desejo de morrer é caracterizado como sendo "o portal" para o comportamento autodestrutivo, durante o modo de vida atual em que o adolescente está vivendo. Os pensamentos suicidas vêm a ser considerados anormais, quando a realização desses pensamentos representa a única saída para os adolescentes (Moreira, 2015).

A ideação suicida refere-se a pensamentos acerca de autodestruição, que compreendem a ideia de que a vida não vale a pena ser vivida, preparando planos específicos para lhe por fim. É apresentada como um indicador fundamental para o risco de suicídio. A presença de ideação suicida é frequentemente um sinal de sofrimento emocional grave e aparece como um dos principais preditores de tentativas de suicídio que se caracterizam como o ato não fatal, que incluem automutilação, auto envenenamento e suicídio consumado (Azevedo, 2014; Silva, 2008).

O suicídio cometido por adolescentes ocorre no mundo inteiro, e está entre as cinco maiores causas de morte na faixa etária entre 15 e 19 anos sendo que, em vários países, ele vem como a primeira ou segunda causa de morte entre adolescentes do sexo masculino e feminino nessa mesma faixa etária. Trata-se de um ato voluntário contra a própria vida, que resulta em morte. Atinge todas as culturas e classes sociais, apresentando uma etiologia multifacetada que vem desde os aspectos biológicos, sociais, genéticos, psicológicos, culturais e ambientais (Moreira, 2015; Silva, 2008).

Em grande parte do mundo o suicídio é estigmatizado e rodeado de tabus. Ao falar que uma pessoa morreu provocase comoção e solidariedade; porém, quando se diz que a morte foi autoprovocada, a fala fica suspensa, praticamente não circula, causando constrangimento. O assunto é evitado ou proibido, ficando uma mácula. De modo geral, a população tende a negar essa atitude, especialmente quando acontece entre os adolescentes e, ainda mais, em crianças. Apesar disso, atualmente sabe-se que os adolescentes são especialmente vulneráveis a reagirem com atitudes suicidas em resposta a conflitos que estejam enfrentando (Abasse, 2009). 
Segundo estudos analisados por Moreira (2015), os principais fatores associados à ideação suicida na adolescência são multifacetados e incluem desde transtornos mentais até as características pessoais do adolescente e de sua estrutura familiar.

Os diferentes contextos que o adolescente esteja inserido podem auxiliar tanto na potencialização quanto na identificação precoce desse comportamento, como por exemplo: a escola, onde o adolescente passa boa parte do seu tempo e estabelece relação com colegas e professores e também a família, pois uma relação familiar próxima favorece acolhimento e atenção aos adolescentes, como um espaço para identificar e prevenir o agravamento do comportamento (Silva, 2017; Fernandes, 2016).

De acordo com Silva (2016) os fatores de risco e de proteção precisam ser analisados e avaliados de forma contextualizada, pois, envolve todo o contexto social, histórico e coletivo do adolescente. Com essa análise, é possível observar que um fator de risco não é limitado apenas em aspectos negativos, ele também pode ser um fator que traz situações de resiliência, resultando uma possível aquisição de habilidades frente as adversidades da vida.

Embora a identificação dos fatores de risco ao suicídio seja importante para a prevenção, os profissionais da saúde devem estar atentos para saber interpretá-los e manejá-los de forma adequada, encaminhando os adolescentes aos serviços de saúde específicos, ou seja, os que estão previstos na Rede de Atenção Psicossocial (RAPS) (Braga, 2013).

A RAPS, instituída pela Portaria MS/GM n 3.088 , de 2011, prevê a criação, a ampliação e a articulação de pontos de atenção à saúde para pessoas com sofrimento ou transtorno mental e com necessidades decorrentes do uso de crack, álcool e outras drogas no âmbito do SUS. Em seus objetivos específicos estão a promoção de cuidados em saúde especialmente a grupos mais vulneráveis, ou seja, crianças, adolescentes, jovens, pessoas em situação de rua e populações indígenas. Um dos serviços essenciais na RAPS é o Centro de Atenção Psicossocial (CAPS), que dentre as suas diferentes modalidades tem o que é destinado ao atendimento ao público infanto-juvenil (CAPSi).

Dessa forma, junto às ações dos profissionais, torna-se necessário o fortalecimento das redes de apoio dos adolescentes, envolvendo principalmente a família, grupos de pares e escola, promovendo relações mais satisfatórias e maior bem-estar, tendo em vista que os relacionamentos pessoais e a percepção de apoio ocupam um importante papel nessa etapa do ciclo vital (Braga, 2013).

Sendo assim, a escolha do presente tema justifica-se pela crescente taxa de suicídio (Fernandes et. al., 2020), tentativas de suicídio e ideação suicida entre os e as adolescentes, caracterizando tal tema como importante problema de saúde pública. Este estudo tem como objetivo identificar entre adolescentes em situação de vulnerabilidade social os que apresentam ideação suicida.

\section{Metodologia}

Estudo de abordagem quantitativa, de corte transversal e descritivo. De acordo com Pereira et al. (2018) a abordagem quantitativa utiliza dados numéricos e grandezas que são analisados por meio de técnicas matemáticas, em que, geram resultados numéricos, aplicáveis aos processos do estudo. O estudo de corte transversal traz de particularidade a observação das variáveis em um único momento e oferece ao pesquisador a observação direta e a realização da coleta de dados em um curto espaço de tempo (Zangirolami-Raimundo; Echeimberg; Leone, 2018). Foram convidados a participar adolescentes de 12 a 18 anos incompletos assistidos por uma Organização Não Governamental (ONG), em Recife/PE. Foram incluídos os jovens com frequência regularizada na instituição e excluídos adolescentes que tivessem deficiência intelectual (previamente identificados pela coordenação pedagógica da instituição) que os impedisse de responder os instrumentos utilizados na pesquisa. 
O período de coleta de dados se deu entre Junho e Agosto de 2017, na própria instituição. Os instrumentos utilizados foram um questionário sociodemográfico com informações relacionadas a idade, sexo, escolaridade, dados familiares e motivos para frequentar a ONG e a Escala de Avaliação do Risco de Suicídio de Columbia (C-SSRS), respondidos pelo próprio adolescente.

A Escala de Avaliação do Risco de Suicídio de Columbia (C-SSRS) trata-se de um instrumento que realiza a avaliação do risco de suicídio através de uma série de perguntas, que se dividem em três sessões a saber: 1) Ideação Suicida, 2) Intensidade da ideação e 3) Comportamento Suicida (Likert Scale). As respostas direcionam a sequência de perguntas seguintes e ajudam a identificar se alguém está em risco de suicídio, avaliar a gravidade e o imediatismo desse risco, bem como o nível de apoio de que a pessoa precisa e as respostas devem ser dadas de acordo com a percepção do indivíduo no momento do preenchimento das questões. No presente estudo, a escala foi aplicada em forma de entrevista pela pesquisadora principal, que recebeu treinamento virtual e tornou-se apta para a função, conforme preconizado pelo autor da escala.

O Fluxograma 1 ilustra a sequência das perguntas. O entrevistado é convidado a responder primeiro a seção de ", Ideação Suicida" (perguntas 1 e 2) se as respostas para ambas forem negativas, segue para a seção " Comportamento Suicida", entretanto, mesmo se o entrevistado responder " não" para a pergunta 2 ele pode ser direcionado para as perguntas 3,4 e 5, a depender da avaliação de necessidade por parte do entrevistador. Se as respostas para as perguntas 1 e 2 forem "' sim" o entrevistado é direcionado aos questionamentos 3, 4, e 5 e, em seguida, para a seção " Intensidade da ideação", na qual as características são avaliadas levando em consideração o tipo de ideação mais intenso, sendo 1 o menos intenso e 5 o mais intenso, perguntando o momento em que ele/ela estava se sentindo com maior tendência suicida. Na seção "Comportamento Suicida" (que deve ser respondida por todos os entrevistados), é marcado um X em todos os itens que se aplicam, caso sejam eventos distintos (Posner, 2009).

Os dados foram analisados descritivamente com auxílio do Software Microsoft Office Excel ® 2013 e expostos em tabelas para facilitar a compreensão.

A pesquisa foi aprovada pelo Comitê de Ética em Pesquisa com seres humanos do Centro de Ciências da Saúde, da UFPE (CEP-CCS) com número do parecer 2.106.776, que contempla os requisitos éticos propostos pela Resolução n466/12 do Conselho Nacional de Saúde. 
Fluxograma 1. Sequência de perguntas para a Escala de Avaliação do Risco de Suicídio de Columbia (C-SSRS).

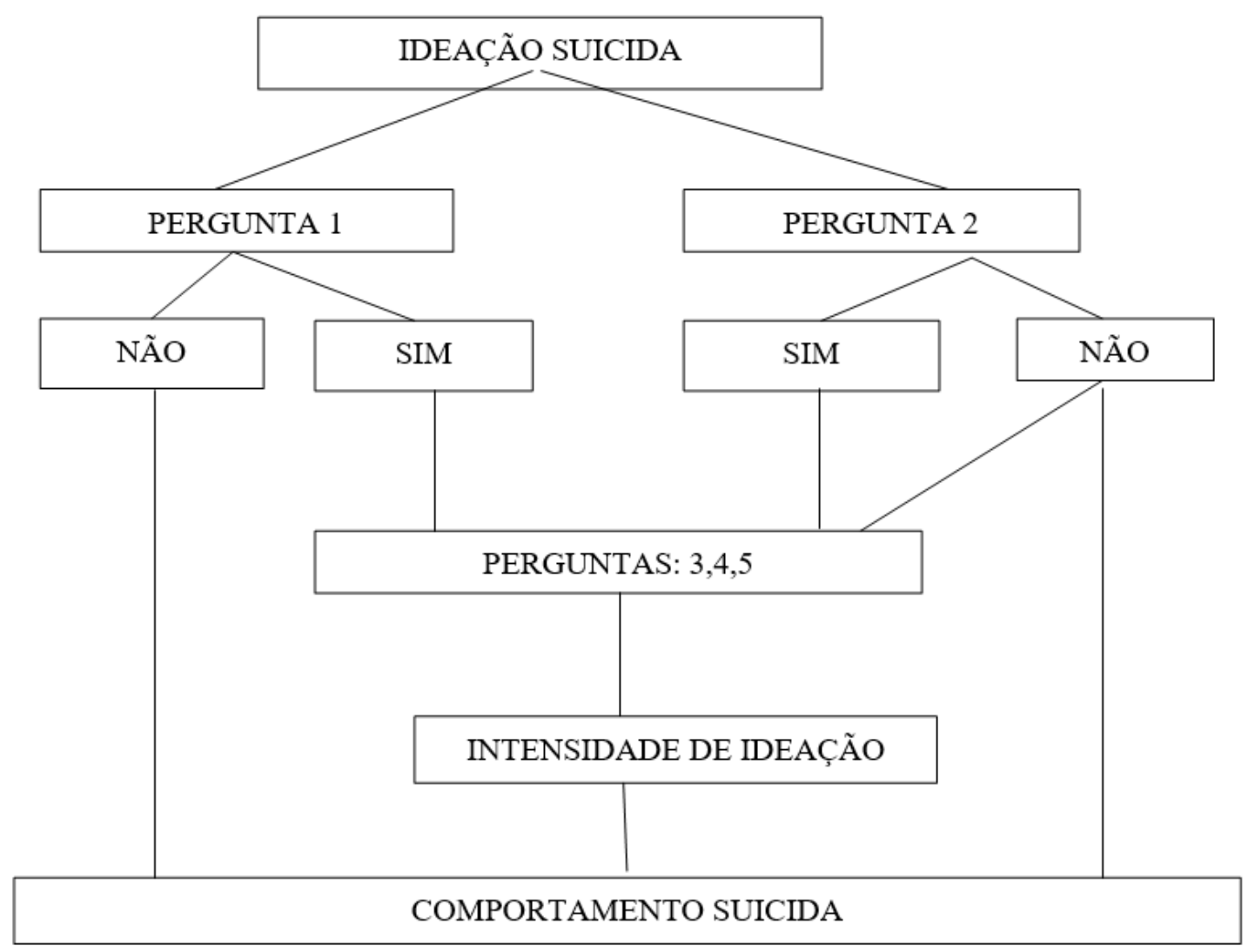

Fonte: Autores (2017).

\section{Resultados}

Participaram do estudo 30 adolescentes na faixa etária entre 12 e 16 anos. Destes, 10 correspondem ao sexo masculino e 20 ao sexo feminino. Para facilitar a compreensão dos achados, os participantes foram listados em ordem alfabética e denominados com a sigla “Ado.”, seguida de um número ordinal crescente.

A maioria dos adolescentes $(n=15)$ está inserida no Ensino Fundamental II, de escola pública. Apenas uma das adolescentes (Ado 9) exerce atividade remunerada informal, como cuidadora, nos horários livres. Todos os adolescentes entrevistados vivem em famílias com média de cinco pessoas, com renda de até um salário mínimo e mais de $50 \%$ dos adolescentes responderam que seus pais são casados ou têm uma união estável. A maior parte dos responsáveis possui ensino fundamental incompleto e $15 \%$ desses estão em situação de desemprego. A maioria dos adolescentes, independente do sexo, vai à ONG sozinho (sem acompanhantes) e frequentam porque os pais os incentivam a realizarem atividades complementares para não ficarem ociosos. A distribuição da amostra quanto às variáveis socioeconômicas e demográficas encontra-se descrita na Tabela 1 . 
Tabela 1 - Caracterização sociodemográfica dos adolescentes.

\begin{tabular}{|c|c|c|c|}
\hline Variáveis & Categorização & $\mathbf{N}^{\circ}$ & $\%$ \\
\hline Sexo & $\begin{array}{l}\text { Masculino } \\
\text { Feminino }\end{array}$ & $\begin{array}{l}10 \\
20\end{array}$ & $\begin{array}{l}33,3 \\
66,7\end{array}$ \\
\hline Idade & $\begin{array}{l}12 \text { anos } \\
13-14 \text { anos } \\
15-16 \text { anos }\end{array}$ & $\begin{array}{r}14 \\
14 \\
2\end{array}$ & $\begin{array}{r}46,7 \\
46,7 \\
6,6\end{array}$ \\
\hline Escolaridade & $\begin{array}{l}4^{\circ} \text { ano }-5^{\circ} \text { ano } \\
6^{\circ} \text { ano- } 7^{\circ} \text { ano } \\
8^{\circ} \text { ano- } 9^{\circ} \text { ano }-1^{\circ} \text { ano } \\
\text { Não estuda }\end{array}$ & $\begin{array}{r}3 \\
15 \\
12 \\
0 \\
\end{array}$ & $\begin{array}{r}10 \\
50 \\
40 \\
0\end{array}$ \\
\hline Estrutura Familiar & $\begin{array}{l}\text { Nuclear } \\
\text { Monoparental } \\
\text { Extensa } \\
\text { Reconstruída }\end{array}$ & $\begin{array}{r}12 \\
5 \\
5 \\
8\end{array}$ & $\begin{array}{l}40 \\
16,67 \\
16,67 \\
26,66\end{array}$ \\
\hline $\mathrm{N}^{\circ}$ de pessoas na casa & $\begin{array}{l}2 \text { à } 3 \text { pessoas } \\
4 \text { à } 5 \text { pessoas } \\
6 \text { à } 7 \text { pessoas } \\
8 \text { à } 9 \text { pessoas }\end{array}$ & $\begin{array}{r}3 \\
16 \\
8 \\
3\end{array}$ & $\begin{array}{l}10 \\
53,4 \\
26,6 \\
10\end{array}$ \\
\hline \multirow{4}{*}{ Renda Familiar } & Sem renda & 0 & 0 \\
\hline & <que 1 salário mínimo & 7 & 11,67 \\
\hline & = 1 salário mínimo & 23 & 83,33 \\
\hline & > que 1 a 2 salários mínimos & 0 & 0 \\
\hline
\end{tabular}

Fonte: Autores (2017).

\subsection{Escala de Avaliação de Risco}

\subsubsection{Seção "Ideação Suicida"}

Com relação as respostas da primeira seção do instrumento (Tabela 2) observou-se que 30 entrevistados $(56,67 \%)$ responderam "NÃO” para a primeira pergunta, enquanto que para a segunda esse índice aumentou para 83,33\%. 
Tabela 2 - Seção "Ideação Suicida", perguntas 1 e 2, indicação do "n” de cada resposta.

\begin{tabular}{|c|c|c|c|c|c|c|}
\hline \multirow{2}{*}{\multicolumn{2}{|c|}{ Questionamento }} & \multirow{2}{*}{$\begin{array}{l}\text { Total de } \\
\text { respondentes }\end{array}$} & \multicolumn{2}{|l|}{ Sim } & \multicolumn{2}{|l|}{ Não } \\
\hline & & & HOMENS & MULHERES & HOMENS & MULHERES \\
\hline 1 & $\begin{array}{l}\text { Você desejou estar } \\
\text { morto/a ou desejou } \\
\text { poder dormir e } \\
\text { nunca mais acordar? }\end{array}$ & 30 & 4 & 9 & 6 & 11 \\
\hline 2 & 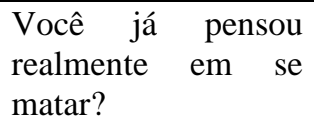 & 30 & 0 & 5 & 10 & 15 \\
\hline
\end{tabular}

Fonte: Autores (2017).

No Fluxograma 2, é apresentado o total de respostas, considerando as possibilidades encontradas nas perguntas 1 e 2 ( 1 a etapa), ou seja: o adolescente responder "SIM" ou "NÃO” apenas para a primeira pergunta, "SIM" ou "NÃO” apenas para a segunda pergunta e "SIM" ou "NÃO" para as duas perguntas. A segunda etapa da seção "ideação suicida" (perguntas 3, 4 e 5) foi realizada com sete adolescentes. Estes foram selecionados da seguinte forma: quatro meninas que responderam "SIM" para os dois questionamentos, uma que respondeu "SIM" somente para o segundo e dois meninos que responderam "SIM" para o primeiro questionamento e "NÃO" para o segundo (Fluxograma 2). Destes, um não avançou para as perguntas 4 e 5 porque, segundo as instruções do instrumento, não haveria necessidade. A distribuição da amostra está descrita no quadro 2.

Fluxograma 2: Seção ideação suicida e número de respondentes, por etapa.

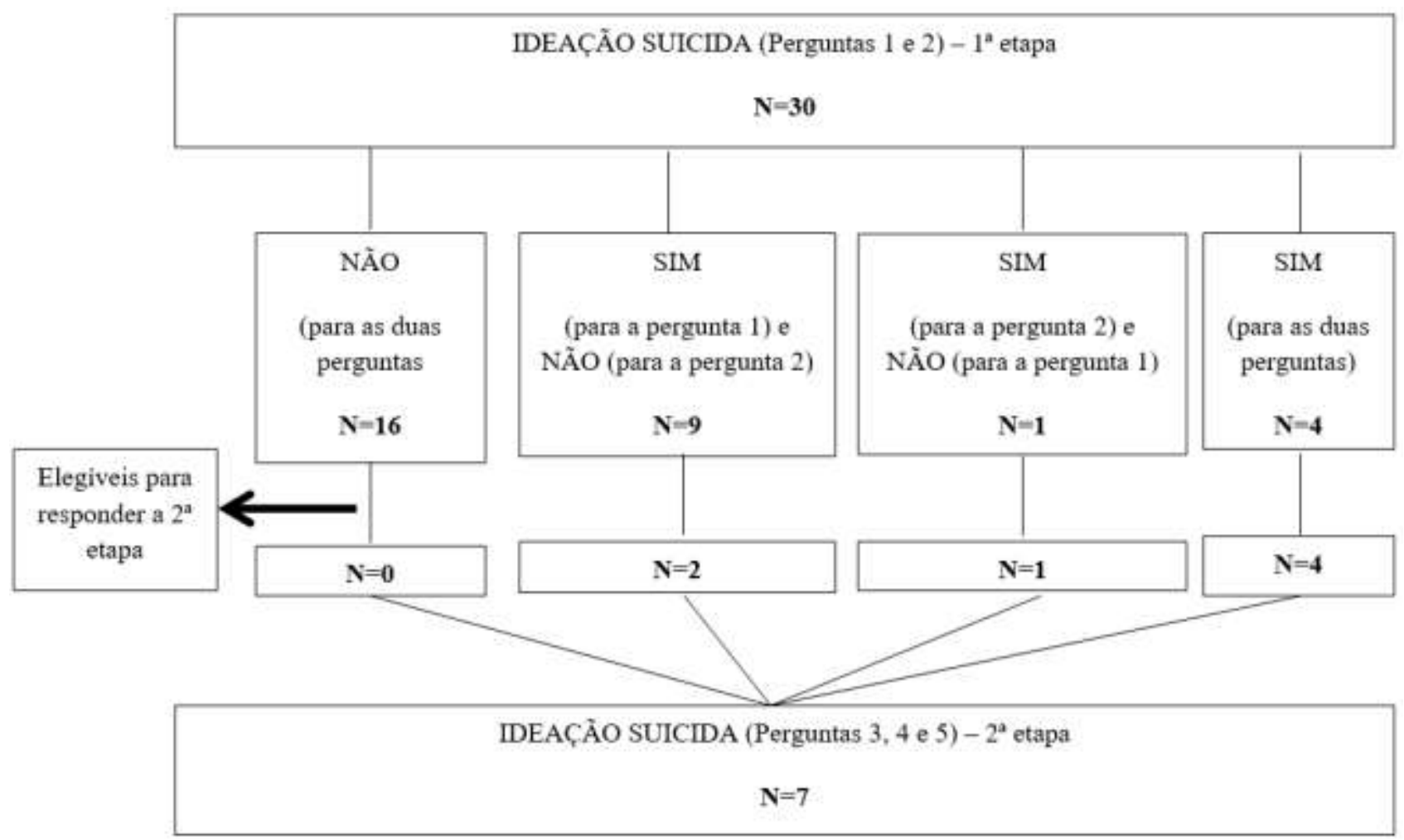

Fonte: Autores (2017). 
Tabela 2 - Seção "Ideação Suicida", perguntas 3, 4 e 5, indicação do "n" de cada resposta.

\begin{tabular}{|c|c|c|c|c|c|}
\hline \multirow{2}{*}{ Questionamento } & \multirow{2}{*}{$\begin{array}{l}\text { Total de } \\
\text { respondentes }\end{array}$} & \multicolumn{2}{|l|}{ Sim } & \multicolumn{2}{|l|}{ Não } \\
\hline & & HOMENS & MULHERES & HOMENS & MULHERES \\
\hline $\begin{array}{l}\text { Ideação suicida ativa } \\
\text { com algum método } \\
\text { (sem plano) sem } \\
\text { intenção de agir }\end{array}$ & 7 & 0 & 2 & 2 & 3 \\
\hline $\begin{array}{l}\text { Ideação suicida ativa } \\
\text { com alguma } \\
\text { intenção de agir, } \\
\text { sem plano } \\
\text { específico. }\end{array}$ & 6 & 0 & 1 & 1 & 4 \\
\hline $\begin{array}{l}\text { Ideação suicida ativa } \\
\text { com plano } \\
\text { específico e intenção }\end{array}$ & 6 & 0 & 1 & 1 & 4 \\
\hline TOTAL & 19 & 0 & 4 & 4 & 12 \\
\hline
\end{tabular}

Fonte: Autores (2017).

\subsubsection{Seção "Intensidade da Ideação"}

Conforme citado anteriormente, apenas cinco meninas (Ado. 1; Ado. 12; Ado. 15; Ado. 23; Ado. 30) responderam a seção sobre intensidade da ideação. Esta seção divide-se em seis questionamentos, em que foram observados os seguintes resultados:

- Maior tendência suicida: apenas uma adolescente (Ado.1) respondeu marcando o nível de intensidade 5, que significa o máximo. As demais não souberam quantificar essa magnitude.

- Frequência de pensamentos suicidas: cada uma das adolescentes respondeu a um nível diferente, variando de "menos de uma vez por semana" até "todos os dias ou quase todos os dias".

- Duração: duas adolescentes (Ado. 23 e Ado. 30) responderam que a duração é "passageira, durando de alguns segundos a minutos" e as outras duas (Ado. 1 e Ado. 12) que durou "menos de uma hora a algum tempo".

- No item sobre controlabilidade: uma adolescente (Ado. 30) respondeu que "é capaz de controlar os pensamentos facilmente"; duas (Ado. 12 e Ado. 23) responderam que "pode controlar os pensamentos com pouca dificuldade"; uma adolescente (Ado. 15) respondeu que "pode controlar os pensamentos com alguma dificuldade"; e uma adolescente (Ado. 1) respondeu que "pode controlar os pensamentos com muita dificuldade".

- Sobre as razões para não cometer suicídio todas as adolescentes acreditam que a família, religião e dor da morte seriam razões que com certeza, as impediriam de cometer o suicídio.

- Levando em considerações as razões para pensar em querer morrer ou se matar: duas adolescentes (Ado.12 e Ado.30) responderam "sobretudo para acabar com o sofrimento (você não conseguia continuar a viver com esse sofrimento ou como você estava se sentindo)"; uma adolescente (Ado.1) responder que: "com certeza para acabar com o sofrimento (você não conseguia continuar a viver com esse sofrimento ou como você estava se sentindo)" e outras duas adolescentes (Ado. 15 e Ado. 23) assinalaram que "não se aplica ao seu caso, por não se identificarem em nenhuma das alternativas". 


\subsubsection{Seção "Comportamento Suicida"}

No item sobre tentativas efetivas os e as adolescentes foram questionados se já cometeram alguma tentativa de suicídio, se fez algo para se ferir ou fez alguma coisa perigosa que poderia ter se matado, apenas adolescentes do sexo feminino responderam que sim, porém $86,67 \%$ negaram ter se engajado em comportamento auto lesivo não suicida.

Franklin et. al, (2017) ressaltam os estágios do comportamento suicida, colocando a ideação quando existe a vontade de tirar a própria vida, o planejamento de como tal fato será realizado e a tentativa, através de ações, que culminam em autoagressões, com objetivo final de morrer. Caso isso ocorra, o suicídio é consumado. Sendo assim, na presente pesquisa, nenhum dos adolescentes apresentou uma tentativa de suicídio interrompida, o que significa dizer que nenhum foi interrompido por uma circunstância externa.

Uma adolescente afirmou ter tido uma tentativa de suicídio abortada, que acontece quando a pessoa começa a dar os primeiros passos em direção a uma tentativa de suicídio, mas para antes de realmente se engajar no comportamento autodestrutivo. Essa mesma adolescente, relata ter tido atos ou comportamentos preparatórios tendo em vista uma tentativa de suicídio, ao reunir medicamentos para realizar ingestão, sendo a única adolescente a apresentar comportamento suicida no período de avaliação e a única a responder a última seção da escala.

\subsubsection{Seção "Letalidade Efetiva"}

Como apenas uma adolescente (Ado. 1) referiu tentativa efetiva de suicídio, esta foi questionada sobre a presença de danos físicos relacionados ao ato. A resposta correspondeu a "ausência de danos físicos ou danos físicos muito leves (escoriações superficiais)"”.

\section{Discussão}

Setti (2011) sugere fatores de risco ao comportamento suicida, tais como: sexo, adolescentes do sexo femininos apresentam maior tentativa suicida em relação aos do sexo masculino; idade, a incidência é aumentada durante a adolescência e a situação socioeconômica também favorece esse comportamento. A Escala de Avaliação de Risco, usa diferentes períodos de avaliação, dependendo da pesquisa ou da necessidade clínica, o período de vida que o adolescente está sendo avaliado. Sendo uma escala oferecem dados para orientar recomendações e tratamentos para prevenir o suicídio (Posner, 2011).

Através dos resultados, após a aplicação da referida escala, mostrou a presença de ideação suicida com maior índice em adolescentes do sexo feminino, que, conforme dito anteriormente, na adolescência as mulheres apresentam taxas mais altas para imaginação e tentativa de suicídio. Com ideação suicida avançando, surge o planejamento suicida, sendo essa a etapa em que o adolescente começa a estabelecer planos de onde, quando e como vão realizar a tentativa (Baggio, 2009). Entretanto, torna-se importante ressaltar que esses fatores individuais não são determinantes para o suicídio e sim as consequências que deles podem ser derivadas.

Além dos fatores supracitados, observa-se que em diferentes estudos existem alguns fatores de riscos gerais que são sempre relatados, como: vulnerabilidade social, família de origem disfuncional, experiências de diversos tipos de violência, condições insalubres de trabalho, uso e abuso de drogas, sexo masculino (Pereira et al., 2018).

Todos os participantes do presente estudo estão inseridos na escola e com rendimento escolar compatível com a idade. Costa (2012) ressalta a importância da escola no processo de construção de identidade e autonomização dos adolescentes, visto que nesse período a escola ganha uma importância maior, principalmente pelo tempo que os adolescentes passam na mesma. Nesse período o adolescente começa a sentir maior responsabilidade e obrigações, dessa forma é 
importante uma articulação entre a escola, pais e/ou responsáveis, a comunidade e outros serviços que fazem parte da Rede de Atenção Psicossocial (Costa, 2012).

A família desempenha um papel importante como agente no processo de socialização, sendo os pais ou principais cuidadores/responsáveis através da socialização primaria responsáveis pela mediação do mundo para a criança. Além disso, a família ocupa outro importante papel na vida do indivíduo, pois ela é o centro da estruturação psíquica. As formas como esses adolescentes se percebem e efetuam as vivências emocionais na família são fontes primárias de importantes informações para a localização de si no seu contexto social (Chechi, 2017).

A estrutura familiar dos entrevistados foi configurada, em sua maioria, do tipo nuclear e extensa. Segundo Borsa (2017) esse modelo de família é composto por pai, mãe e filhos, em que, a mulher ocupa fundamentalmente um papel de maternidade e desempenho de várias funções no ambiente familiar, para o desenvolvimento desse arranjo familiar. Polli (2012) fala sobre as definições das famílias extensas, são aquelas na qual as figuras familiares extrapolam a família nuclear, pois, estão inclusos os avós, tios (as), primos (as), sendo essa inclusão uma medida de apoio aos cuidados dos adolescentes ou até mesmo de uma assistência financeira. O conceito de família atualmente perpassa também por outros modelos, não necessariamente está ligada através do casamento de um homem e uma mulher. Onde existir pessoas ligadas, seja por laço sanguíneo ou não, mas por laços afetuosos, haverá uma família, encaixando-se também o modelo de família homoafetiva (Poli; Poli, 2013).

De Lima e Braga (2013) apontam que a vulnerabilidade gerada por situações de baixo nível econômico é uma situação social que pode predispor ao suicídio, já que o desemprego pode acarretar em uma situação de instabilidade familiar gerando situações de alta ansiedade por parte dessa família. As famílias dos entrevistados têm renda de até um salário mínimo apresentando assim um baixo nível econômico. A situação dos pais dos adolescentes se destaca entre desempregados, do lar ou autônomo, o que vem a interferir na renda familiar.

Em situações de instabilidade financeira o trabalho precoce entre adolescentes na rotina familiar é comum, visto como uma possibilidade de melhora financeira e quebra do ócio. Entre os entrevistados, uma adolescente, relatou exercer atividade remunerada não-formal, como cuidadora de uma prima (criança), essa ação resulta em impactos que podem ser gerados com esse trabalho precoce devido à alta responsabilidade que a função pode gerar, como: desequilíbrio emocional, fadiga e falta de tempo para se engajar em atividades escolares (evasão escolar, índices de reprovação) e complementares (prática de esportes, brincar) (Campos, 2003).

Porém, os adolescentes podem desempenhar uma atividade laborativa, desde que dentro do que é previsto em lei, como o Programa de Menor Aprendiz, que é destinado a adolescentes com menos de dezesseis anos de idade proibindo que o mesmo exerça atividades insalubres, perigosas, noturnas e com longas jornadas de trabalhos entre outras (Silva, 2010). Dentre os benefícios estão, segundo Coelho (2015) que o empregador é obrigado a conceder ao menor o tempo necessário para frequentar as aulas, inscrição do menor em programas que garante uma formação técnico-profissional, entre outros que garantem a segurança física e psíquica dos adolescentes.

Além de atividades remuneradas, no contraturno escolar, a frequência dos adolescentes em atividades complementares de ONG's auxilia não apenas na quebra do ócio, mas também, os conscientiza sobre seus direitos e deveres e até para o exercício da autonomia (Dias; Pereira, 2016). Cunha (2013) relata que a participação nessas atividades complementares também recebe influência dos pais, em que, muitas vezes precisam se ausentar durante o dia devido a necessidade de trabalhar e confiam na capacidade desses locais em proporcionar um auxílio benéfico no desenvolvimento dos seus filhos.

Na seção de Intensidade de Ideação as adolescentes, em média, afirmam que os pensamentos permeiam entre duas a cinco vezes por semana, com pouca durabilidade e apresentando pouca dificuldade para controlar a permanência desses 
pensamentos. Elas ainda afirmaram que, dentre as razões para não cometer o suicídio, estão à família e a religião que as impediriam de colocar suas ações em prática. Martins, Trindade e Almeida (2003), afirmam que embora os adolescentes precisem de espaço para ter liberdade para se divertir, eles precisam também de apoio e proteção para se sentirem seguros, sendo assim o apoio e a proteção são elementos significativos para a vida e são colocados em agentes externos, como: pais, escola, amigos, entre outros fatores de proteção.

Quanto às razões para cometer o suicídio, as adolescentes afirmaram acreditar que suas motivações são reais e explicariam qualquer tentativa. Esse dado corrobora com o estudo de Borges (2009), o qual afirma que a intenção de querer morrer pode representar um início de renúncia para lutar contra uma angústia intolerável, podendo ocorrer, muitas vezes, por falta de expectativas positivas em pessoas mais desesperançosas ou depressivas, com tendências a superestimar as dificuldades e a resolução de problemas.

Abreu (2010) se refere ao comportamento suicida como um ato de autoagressão bem como variantes relacionadas às tentativas de suicídio, com alta ou baixa letalidade que vem a ocorrer dentro de um contexto social. Nesse estudo, a maioria dos entrevistados afirmou ter se envolvido em algum comportamento autolesivo não suicida, porém uma entrevistada, com os pais separados, residindo apenas com a mãe, afirmou ter tido atos ou comportamentos preparatórios tendo em vista uma tentativa de suicídio, ao reunir medicamentos para realizar ingestão. Essa ação da adolescente teve baixa letalidade, com ausência de danos físicos.

A escolha dos meios para tentativa de suicídio, através de ingestão medicamentosa, é explicada na literatura como sendo uma das medidas mais comuns, pois esses produtos não apresentam controle suficiente na sua comercialização, além dos pais não serem orientados a dificultar o acesso aos medicamentos que existem nas suas residências (Avanci, 2005).

Os resultados gerais deste estudo foram encaminhados para o setor de psicologia da ONG, para orientação e sugestão de abordagem da temática. Apenas o caso da adolescente que fez a pontuação máxima da escala foi encaminhado com os dados completos, visto que já estava previsto no projeto. A identificação precoce de adolescentes em situação de risco para o suicídio é desafiadora, pois consiste no reconhecimento das pessoas em situação de vulnerabilidade, perceber quais situações influenciam para o comportamento suicida além de estruturar intervenções eficazes. Dentre as intervenções estão os encaminhamentos para serviços especializados, que podem ser de âmbito privado ou público, e nesse caso, os que são inseridos na Rede de Atenção Psicossocial, especialmente os Centros de Atenção Psicossocial (CAPS), além do próprio suporte oferecido pela ONG, que trabalha na prevenção do suicídio e funciona como suporte e retaguarda para as ações dos demais serviços (Conte, 2012; OMS, 2002).

Um fator protetor em relação ao risco suicida é a rede de suporte desses adolescentes, que inclui: família, a escola e até mesmo a ONG a qual eles frequentam. Esses suportes são vistos pela literatura como sendo fatores de proteção, pois fazem os adolescentes perceberem que são queridos, amados, favorecendo assim a melhora da autoestima, sendo considerado um processo recíproco, levando-se em consideração que nesses ambientes, existem uma troca entre quem oferece o apoio e quem recebe, formando assim uma rede de apoio social (Gaspari; Botega, 2002).

\section{Conclusão}

O presente estudo teve como foco identificar os adolescentes com ideação suicida considerando, diversos fatores que aumentam o risco de adolescente vir a cometer um ato autolesivo, sugere-se de identificar fatores de riscos, como as privações afetivas e socioeconômicas, que podem expor os adolescentes a situação de riscos para a ideação. Em presença dos resultados e discussões, foi possível refletir que a situação de vulnerabilidade social que se encontram os adolescentes, observados através de análise dos dados socioeconômicos os coloca em situação de risco para predisposição a ideação suicida. 
Apesar da condição dos de vulnerabilidade social, os achados da Escala de Avaliação e Risco revelaram que pouco mais da metade dos adolescentes entrevistados, não apresentaram ideação suicida, o que pode estar associado ao suporte familiar, escolar e da ONG. Ainda assim, uma boa parte dos adolescentes entrevistados apresentaram respostas que afirmam o desejo de estar morto, podendo esses pensamentos evoluírem para uma tentativa efetiva ao longo da vida, portanto, precisam ser investigados e acompanhados por equipes articuladas e especializada.

Frente à gravidade da temática, é necessário pensar em intervenções breves e eficazes que possam detectar precocemente a presença de ideação suicida nos adolescentes e principalmente em intervenções que envolvam todos os atores, ou seja, os próprios adolescentes, familiares, escola, comunidade e tanto profissionais de saúde, quanto os de outras áreas. Por se tratar de um problema de saúde pública, ainda é necessário que exista treinamento e suporte para os profissionais adquirirem manejos para acolher esse adolescente juntamente com a sua família, facilitar e encaminhar os casos a serviços especializados de saúde, dessa forma, intervindo precocemente na ideação suicida, impedindo o comportamento sem concretizar o suicídio.

A utilização da Escala de Avaliação de Risco permite a análise de forma isolada tanto da presença de ideação quanto ao risco de suicídio de forma nivelada e possibilita a distinção do comportamento. Entretanto, por ser um instrumento com questionamentos amplos e algumas respostas preestabelecidas, sugere-se o uso desse acompanhado de uma investigação mais ampla, combinando com outras formas avaliativas e de acompanhamento.

Considerando a complexidade do tema relacionado ao suicídio, sugere-se que outras pesquisas, envolvendo diferentes estratégias metodológicas e um número maior de participantes, possam ser adotadas com o intuito de diversificar os olhares sobre o tema, visando a adoção de estratégias eficazes para a prevenção do suicídio na adolescência.

\section{Referências}

Abasse, M. L. F., Oliveira, R. C. D., Silva, T. C. \& Souza, E. R. D. (2009). Análise epidemiológica da morbimortalidade por suicídio entre adolescentes em Minas Gerais, Brasil. Ciência \& Saúde Coletiva, 14(2), 407-16.

Abreu, K. P. D., Lima, M. A. D. D. S., Kohlrausch, E. R., \& Soares, J. D. S. F. (2010). Comportamento suicida: fatores de risco e intervenções preventivas. Revista Eletrônica de Enfermagem. Goiânia. 12(1), 195-200.

Araújo, L. D. C., Vieira, K. F. L. \& Coutinho, M. D. P. D. L. (2010). Ideação suicida na adolescência: um enfoque psicossociológico no contexto do ensino médio. Psico-USF, 15(1), 47-57.

Arruda Silva, M. F. \& Siqueira, A. C. (2017). O perfil de adolescentes com comportamentos de autolesão identificados nas escolas estaduais em Rolim de Moura-RO. Revista farol, 3(3), 5-20.

Avanci, J. Q., Assis, S. G., Oliveira, R. V., Ferreira, R. M. \& Pesce, R. P. (2007). Fatores associados aos problemas de saúde mental em adolescentes. Psicologia: Teoria e Pesquisa, 23(3), 287-294.

Avanci, R. D. C., Pedrão, L. J. \& Costa Júnior, M. L. D. (2005). Perfil do adolescente que tenta suicídio em uma unidade de emergência. Revista brasileira de Enfermagem, 58(5), 535-539.

Azevedo, A. \& Matos, A. P. (2014). Ideação suicida e sintomatologia depressiva em adolescentes. Psicologia, Saúde \& Doenças, 15(1), 179-190.

Baggio, L., Palazzo, L. S. \& Aerts, D. R. G. D. C. (2009). Planejamento suicida entre adolescentes escolares: prevalência e fatores associados. Cadernos de Saúde Pública, 25, 142-150.

Baptista, M. N., Baptista, A. S. D. \& Dias, R. R. (2001). Estrutura e suporte familiar como fatores de risco na depressão de adolescentes. Psicologia: ciência e profissão, 21(2), 52-61.

Borges, V. R. \& Werlang, B. S. G. (2006). Estudo de ideação suicida em adolescentes de 15 a 19 anos. Estudos de Psicologia (Natal), $11(3), 345-351$.

Borsa, J. C. \& Nunes, M. L. T. (2017). Aspectos psicossociais da parentalidade: o papel de homens e mulheres na família nuclear. Psicologia Argumento, 29(64).

Brasil, M. (2014). Atenção psicossocial a crianças e adolescentes no SUS: tecendo redes para garantir direitos. Ministério da Saúde, Conselho Nacional do Ministério Público.

Campos, H. R. \& Francischini, R. (2003). Trabalho infantil produtivo e desenvolvimento humano. Psicologia em Estudo, 8(1), 119-129. 
Chechi, P. \& Dias, A. C. G. (2003). Comunicação em famílias com filhos adolescentes. Disciplinarum Scientia Saúde, 4(1), 143-156.

Coelho, M. D. R. P. \& Alves, D. O. I. M. (2015). O menor aprendiz: contrato de aprendizagem e o desenvolvimento social nas relações de trabalho. Revista Científica da FACERB, Rio Bonito, 2 (1), 21-44.

Conte, M., Meneghel, S. N., Trindade, A. G., Ceccon, R. F., Hesler, L. Z., Cruz, C. W. \& Jesus, I. (2012). Programa de Prevenção ao Suicídio: estudo de caso em um município do sul do Brasil. Ciência \& Saúde Coletiva, 17, 2017-2026. \& Saúde Coletiva, 17, 2017-2026.

Costa, I. A. N. C. D. (2012). Adolescência: Ideação suicida, depressão, desesperança e memórias autobiográficas (Doctoral dissertation, ISPA-Instituto Universitário das Ciências Psicológicas, Sociais e da Vida).

Cunha, A. D. J. D. (2013). A importância das atividades extracurriculares na motivação escolar e no sucesso escolar (Doctoral dissertation, [sn]).

Dias, C. N. \& Pereira, E. R. (2016). Hip Hop na ONG: os sentidos produzidos por crianças e adolescentes em oficinas de danças. Horizonte Científico, 10 (1), $1-18$.

Eisenstein, E. (2005). Adolescência: definições, conceitos e critérios. Adolescência e saúde, 2(2), 6-7.

Fernandes, F. Y., Freitas, B. H. B. M. D., Marcon, S. R., Arruda, V. L. D., Lima, N. V. P. D., Bortolini, J. \& Gaíva, M. A. M. (2020). Tendência de suicídio em adolescentes brasileiros entre 1997 e 2016. Epidemiologia e Serviços de Saúde, 29, e2020117.

Franklin, J. C., Ribeiro, J. D., Fox, K. R., Bentley, K. H., Kleiman, E. M., Huang, X. \& Nock, M. K. (2017). Risk factors for suicidal thoughts and behaviors: a meta-analysis of 50 years of research. Psychological bulletin, 143(2), 187.

Merten Fernandes, V. \& Grasel Zacharias, D. (2016). Adolescência, suicídio e redes de apoio: o desejo de morte enquanto grito pela vida. Jornada de Pesquisa em Psicologia.

Muza, G. M., Bettiol, H., Muccillo, G. \& Barbieri, M. A. (1997). Consumo de substâncias psicoativas por adolescentes escolares de Ribeirão Preto, SP (Brasil). I-Prevalência do consumo por sexo, idade e tipo de substância. Revista de saúde pública, 31(1), 21-29.

Gaspari, V. P. P., Botega, N. J. (2002). Rede de apoio social e tentativa de suicídio. J. bras. psiquiatr, 51(4), $233-240$.

Lima Braga, L. \& Dell'Aglio, D. D. (2013). Suicídio na adolescência: fatores de risco, depressão e gênero. Contextos Clínicos, 6(1), 2-14.

Martins, P. D. O.; Trindade, Z. A. \& Almeida, Â. M. D. O. (2003). O ter e o ser: representações sociais da adolescência entre adolescentes de inserção urbana e rural. Psicologia: Reflexão e crítica, 16(3), 555-568.

Moreira, L. C. D. O. \& Bastos, P. R. H. D. O. (2015). Prevalência e fatores associados à ideação suicida na adolescência: revisão de literatura. Psicologia Escolar e Educacional, 19(3), 445-453.

World Health Organization (WHO). (2006). Prevenção do suicídio: Um recurso para conselheiros. Genebra: OMS. http://www. who. int/mental_health/media/counsellors_portuguese. pdf.

Organização Mundial da Saúde (OMS). (2012). Ação de saúde pública para a prevenção de suicídio: uma estrutura.

Pereira, A. S., Willhelm, A. R., Koller, S. H. \& Almeida, R. M. M. D. (2018). Fatores de risco e proteção para tentativa de suicídio na adultez emergente. Ciência \& Saúde Coletiva, 23, 3767-3777.

Pereira, A. S. et al. (2018). Metodologia da pesquisa científica. UFSM. https://repositorio.ufsm.br/bitstream/handle/1/15824/Lic_Computacao_MetodologiPesquisa-Cientifica.pdf?sequence $=1$.

Pessalacia, J. D. R., Menezes, E. S. D. \& Massuia, D. (2010). A vulnerabilidade do adolescente numa perspectiva das políticas de saúde pública. Bioethikos, 4(4), 423-30.

Poli, L. C. \& Poli, L. M. A família contemporânea: reflexões sobre o casamento homoafetivo à luz dos princípios constitucionais. Nomos: Revista do Programa de Pós-Graduação em Direito da UFC, 33(1).

Polli, R. G. \& Arpini, D. M. (2012). O olhar de meninos de grupos populares sobre a família. Estudos de Psicologia (Campinas), 29(4), 531-540.

Posner, K., Brown, G. K., Stanley, B., Brent, D. A., Yershova, K. V., Oquendo, M. A. \& Mann, J. J. (2011). The Columbia-Suicide Severity Rating Scale: initial validity and internal consistency findings from three multisite studies with adolescents and adults. American journal of psychiatry, $168(12), 1266-1277$.

Quiroga, F. L. \& Vitalle, M. S. D. S. (2013). O adolescente e suas representações sociais: apontamentos sobre a importância do contexto histórico. Physis: revista de saúde coletiva, 23, 863-878.

Rodrigues, M. E. D. S., Silveira, T. B. D., Jansen, K., Cruzeiro, A. L. S., Ores, L., Pinheiro, R. T. \& Souza, L. D. D. M. (2012). Risco de suicídio em jovens com transtornos de ansiedade: estudo de base populacional. Psico-USF, 17(1), 53-62.

Schoen-Ferreira, T. H., Aznar-Farias, M. \& Silvares, E. F. D. M. (2010). Adolescência através dos séculos. Psicologia: Teoria e Pesquisa, $26(2), 227-234$.

Setti, L. A. P. (2011). Suicídio: uma reflexão discursiva e as possibilidades de ações preventivas a partir dos fatores de riscos. Universidade do Vale do Itajaí (UNIVALI). Programa de Pós-Graduação Stricto Sensu em Saúde Mestrado Profissional em Saúde e Gestão do Trabalho. Itajaí, Santa Catarina, SC, Brasil.

Silva, A. M. B. \& Enumo, S. R. F. (2016). Teses e Dissertações Brasileiras Sobre Fatores de Risco e Proteção, Vulnerabilidade e Resiliência na Adolescência. Revista Brasileira Adolescência e Conflitualidade, (14), 13-20. 
Research, Society and Development, v. 10, n. 2, e48410212808, 2021

(CC BY 4.0) | ISSN 2525-3409 | DOI: http://dx.doi.org/10.33448/rsd-v10i2.12808

Silva, M. N. (2010). A exploração do trabalho da criança e do adolescente e o contrato de trabalho do menor-aprendiz em conformidade com a CLT e a garantia do acesso à educação. Conteúdo Jurídico, Brasília-DF, 17.

Silva, R. G. D. (2008). Ideação suicida: A influência da vinculação e do bem-estar psicológico (Doctoral dissertation).

Teixeira, C. M. F. S. (2002). A escola como espaço de prevenção ao suicídio de adolescentes-relato de experiência. Revista Inter Ação, 27(1), 99-114.

Zangirolami-Raimundo, J., Echeimberg, J. O. \& Leone, C. (2018). Tópicos de metodologia de pesquisa: Estudos de corte transversal. J Hum Growth Dev, 28(3), 356-60. 